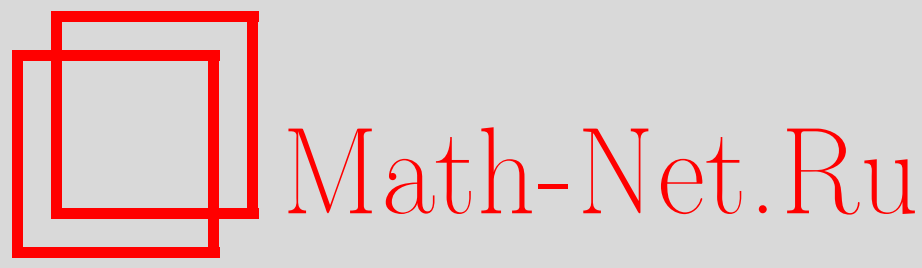

В. И. Афанасьев, Функциональные предельные теоремы для высокоуровневых докритических ветвящихся процессов в случайной среде, Дискрет. матем., 2014, том 26, выпуск 2, 6-24

DOI: https://doi.org/10.4213/dm1277

Использование Общероссийского математического портала Math-Net.Ru подразумевает, что вы прочитали и согласны с пользовательским соглашением http: //www . mathnet.ru/rus/agreement

Параметры загрузки:

IP : 52.87 .193 .239

26 апреля 2023 г., $17: 37: 24$

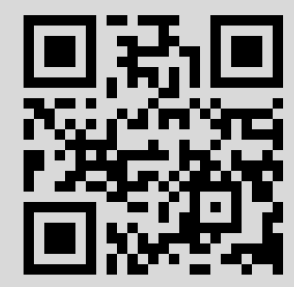




\title{
Функциональные предельные теоремы для высокоуровневых докритических ветвящихся процессов в случайной среде
}

\author{
() 2014 г. В. И. Афанасьев*
}

Рассматривается докритический ветвящийся процесс в случайной среде. Предполагается, что производящая функция моментов шага сопровождающего случайного блуждания принимает значение 1 при некотором положительном значении аргумента. Установлены функциональные предельные теоремы для численностей различных поколений и для времен достижения различных уровней.

Ключевые слова: ветвящийся процесс в случайной среде, достижение высокого уровня, условные предельные теоремы.

1. Пусть $\left\{\xi_{n}, n \in \mathbb{N}_{0}\right\}$ - ветвящийся процесс в случайной среде (ВПСС), задаваемый последовательностью независимых одинаково распределенных (случайных) производящих функций $\left\{f_{n}(s), n \in \mathbb{N}\right\}$. Определение этого процесса можно найти в [1]. Отметим, что $\xi_{n}$ - численность $n$-го поколения (считаем, что $\xi_{0}=1$ ). Производящая функция $f_{n}(s), s \in[0,1]$, определяет закон размножения частиц в $(n-1)$-м поколении, $n \in \mathbb{N}$.

Положим при $i \in \mathbb{N}$

$$
X_{i}=\ln f_{i}^{\prime}(1), \quad \eta_{i}=\frac{f_{i}^{\prime \prime}(1)}{\left(f_{i}^{\prime}(1)\right)^{2}}
$$

(при этом считаем, что $0<f_{1}^{\prime \prime}(1)<+\infty$ п.н.). Заметим, что случайные векторы $\left(X_{1}, \eta_{1}\right),\left(X_{2}, \eta_{2}\right), \ldots$ являются независимыми и одинаково распределенными. Введем сопровождающее случайное блуждание:

$$
S_{0}=0, \quad S_{n}=\sum_{i=1}^{n} X_{i}, n \in \mathbb{N} .
$$

Всюду в дальнейшем полагаем, что распределение случайной величины $X_{1}$ является неарифметическим.

Предположим, что процесс $\left\{\xi_{n}\right\}$ является докритическим, т.е. $\mathbf{E} X_{1}<0$, и существует такое положительное число $\varkappa$, что

$$
\mathbf{E} \exp \left(\varkappa X_{1}\right)=1, \quad \mathbf{E}\left(\left|X_{1}\right| \exp \left(\varkappa X_{1}\right)\right)<+\infty
$$

* Место работы: Математический институт им. В.А. Стеклова РАН, e-mail: viafan@mi.ras.ru

Работа выполнена при поддержке РФФИ, проект 11-01-00139. 
Условие (1) касается случайного блуждания $\left\{S_{n}\right\}$, которое в случае докритического ВПСС имеет отрицательный снос. Это условие является классическим для случайных блужданий с отрицательным сносом и позволяет осуществить переход к сопряженному случайному блужданию с положительным сносом.

Кроме того, предположим, что

$$
\mathbf{E}\left(\xi_{1} \ln \left(\xi_{1}+1\right) \exp \left((\varkappa-1) X_{1}\right)\right)<+\infty,
$$

а при $\varkappa \geqslant 1$ существует такое число $p>\varkappa$, что

$$
\mathbf{E}\left(\xi_{1}^{p} \exp \left((\varkappa-p) X_{1}\right)\right)<+\infty .
$$

Для процесса $\left\{\xi_{n}\right\}$ введем момент первого достижения уровня $x>1$ :

$$
T_{x}=\min \left\{n: \xi_{n}>x\right\},
$$

и время жизни:

$$
T=\min \left\{n: \xi_{n}=0\right\} .
$$

В [2] установлено, что если процесс $\left\{\xi_{n}\right\}$ является докритическим и выполнены условия (1)-(3), то при $x \rightarrow+\infty$

$$
\mathbf{P}\left(T_{x}<+\infty\right) \sim c_{0} x^{-\varkappa},
$$

где $c_{0}-$ положительная постоянная. Положим

$$
a=\mathbf{E}\left(X_{1} \exp \left(\varkappa X_{1}\right)\right) .
$$

В [3] установлены законы больших чисел для случайных величин $T_{x}$ и $T$. Оказывается, если выполнены условия (1)-(3), то при $x \rightarrow+\infty$

$$
\left\{\frac{T_{x}}{\ln x} \mid T_{x}<+\infty\right\} \stackrel{\mathbf{P}}{\rightarrow} \frac{1}{a} ;
$$

если, к тому же, $\mathbf{E}\left(\ln ^{+} \eta_{1}\right)^{1+\delta}<+\infty$ при некотором $\delta>0$, то

$$
\left\{\frac{T}{\ln x} \mid T_{x}<+\infty\right\} \stackrel{\mathbf{P}}{\rightarrow} \frac{1}{a}-\frac{1}{\mathbf{E} X_{1}} .
$$

В добавление к условиям (1)-(3) предположим, что

$$
\mathbf{E}\left(X_{1}^{2} \exp \left(\varkappa X_{1}\right)\right)<+\infty .
$$

Положим $\sigma^{2}=\mathbf{E}\left(X_{1}^{2} \exp \left(\varkappa X_{1}\right)\right)-a^{2}$. Очевидно, что $0<\sigma^{2}<+\infty$.

Пусть $B=\{B(t), t \in[0,1]\}$ - стандартное броуновское движение. В настоящей работе устанавливается функииональная предельная теорема для времен достижения различных уровней.

Теорема 1. Пусть $\left\{\xi_{n}, n \in \mathbb{N}_{0}\right\}$ - докритический ветвящийся процесс в случайной среде и выполнены условия (1)-(4). Тогда при $x \rightarrow+\infty$

$$
\left\{\frac{T_{x^{t}}-t \ln x / a}{\sigma a^{-3 / 2} \sqrt{\ln x}}, t \in[0,1] \mid T_{x}<+\infty\right\} \stackrel{D}{\rightarrow} B,
$$

где символ $\stackrel{D}{\rightarrow}$ означает сходимость по распределению в пространстве $D[0,1]$ c топологией Скорохода. 
Кроме того, устанавливается функииональная предельная теорема для численностей различных поколений.

Теорема 2. Пусть $\left\{\xi_{n}, n \in \mathbb{N}_{0}\right\}$ - докритический ветвящийся процесс в случайной среде и выполнены условия (1)-(4). Тогда при $у \rightarrow+\infty$

$$
\left\{\frac{\ln \xi_{\lfloor t y / a\rfloor}-t y}{\sigma \sqrt{y / a}}, t \in[0,1) \mid T_{\exp y}<+\infty\right\} \stackrel{D}{\rightarrow} B .
$$

Отметим, что в теореме 2 переменная $t$ принадлежит промежутку $[0,1)$, а не отрезку $[0,1]$. Сходимость в этой теореме следует понимать как сходимость по распределению в пространстве $D[0, u]$ для произвольного фиксированного $u \in(0,1)$.

Соответствующие результаты для критического ветвящегося процесса в случайной среде установлены в работах [4]-[6].

2. Предположим, что выполнены условия (1)-(3). Изложим некоторые вспомогательные результаты, полученные в [2]. Обозначим $\Delta$ совокупность всех вероятностных мер на множестве целых неотрицательных чисел. Введем на $\Delta$ метрику полной вариации, относительно которой $\Delta$ является полным сепарабельным метрическим пространством. Пусть производящей функции $f_{n}(s)$ соответствует вероятностная мера $Q_{n}$.

Перейдем к новой вероятностной мере $\widetilde{\mathbf{P}}$ и соответствующему ей математическому ожиданию $\widetilde{\mathbf{E}}$, полагая для любого $n \in \mathbb{N}$ и любой измеримой ограниченной числовой функции $g$, заданной на множестве $\Delta^{n} \times \mathbb{N}_{0}^{n}$,

$$
\widetilde{\mathbf{E}} g\left(Q_{1}, \ldots, Q_{n} ; \xi_{1}, \ldots, \xi_{n}\right)=\mathbf{E}\left(\exp \left(\varkappa S_{n}\right) g\left(Q_{1}, \ldots, Q_{n} ; \xi_{1}, \ldots, \xi_{n}\right)\right) .
$$

Относительно этой меры последовательность $\left\{\xi_{n}\right\}$ является надкритическим ВПСС, при этом в качестве среды следует рассматривать $\left\{Q_{n}\right\}$. Отметим, что $\widetilde{\mathbf{E}} X_{1}=a$.

Последовательность $\left\{\xi_{n} / \exp \left(S_{n}\right)\right\}$, рассматриваемая при фиксированной случайной среде, является неотрицательным мартингалом, поэтому $\widetilde{\mathbf{P}}$-п.н. существует

$$
\lim _{n \rightarrow \infty} \frac{\xi_{n}}{\exp \left(S_{n}\right)}=W<+\infty .
$$

Введем случайные события

$$
\begin{gathered}
D=\left\{\lim _{n \rightarrow \infty} \xi_{n}=+\infty\right\}, D^{*}=\{W>0\}, \quad G_{k}=\left\{\xi_{k}>0\right\}, \quad D_{x}=\left\{T_{x}<+\infty\right\}, \\
D_{x, k}=D \cap D_{x} \cap\left\{T_{x}>k, \sup _{n: n>k}\left|\frac{\exp \left(S_{n}\right)}{\xi_{n}}-\frac{\exp \left(S_{k}\right)}{\xi_{k}}\right| \leqslant \varepsilon \frac{\exp \left(S_{k}\right)}{\xi_{k}}\right\},
\end{gathered}
$$

где $k \in \mathbb{N}$, а $\varepsilon \in(0,1 / 2)$. Оказывается, $\widetilde{\mathbf{P}}(D)>0, \widetilde{\mathbf{P}}\left(D \triangle D^{*}\right)=0$ и

$$
\begin{gathered}
\lim _{x \rightarrow+\infty} \widetilde{\mathbf{P}}\left(D \triangle D_{x}\right)=0, \\
\lim _{k \rightarrow \infty} \widetilde{\mathbf{P}}\left(D \triangle G_{k}\right)=0, \\
\lim _{k \rightarrow \infty} \limsup _{x \rightarrow+\infty} \widetilde{\mathbf{P}}\left(D_{x} \triangle D_{x, k}\right)=0, \\
\lim _{k \rightarrow \infty} \limsup _{x \rightarrow+\infty} \widetilde{\mathbf{P}}\left(G_{k} \triangle D_{x, k}\right)=0 .
\end{gathered}
$$


Отметим также, что семейство случайных величин $\left\{\left(\xi_{T_{x}} / \exp \left(S_{T_{x}}\right)\right)^{\varkappa}, x>1\right\}$ равномерно интегрируемо относительно меры $\widetilde{\mathbf{P}}$ (полагаем $\xi_{T_{x}} / \exp \left(S_{T_{x}}\right)=W$ при $\left.T_{x}=+\infty\right)$.

3. Приступим к доказательству теоремы 1 . Для каждого $k \in \mathbb{N}$ введем случайное блуждание

$$
S_{n}^{(k)}=S_{n+k}-S_{k}, \quad n \in \mathbb{N}_{0}
$$

Положим при $y>0$

$$
\mathcal{T}_{y}^{(k)}=\min \left\{n: S_{n}^{(k)}>y\right\}, k \in \mathbb{N}_{0} .
$$

Если событие $D_{x, k}$ произошло, то при всех $n>k$

$$
\frac{1}{1+\varepsilon} \frac{\xi_{k}}{\exp S_{k}} \leqslant \frac{\xi_{n}}{\exp S_{n}} \leqslant \frac{1}{1-\varepsilon} \frac{\xi_{k}}{\exp S_{k}}
$$

и, следовательно,

$$
S_{n-k}^{(k)}+\beta_{1} \leqslant \ln \xi_{n} \leqslant S_{n-k}^{(k)}+\beta_{2}
$$

где

$$
\beta_{1}=\ln \xi_{k}-\ln (1+\varepsilon), \quad \beta_{2}=\ln \xi_{k}-\ln (1-\varepsilon) .
$$

Это означает, что если событие $D_{x, k}$ произошло и, к тому же, $T_{x^{t}}>k$ и $\ln x>\beta_{2}$, то

$$
\mathcal{T}_{\ln x-\beta_{2}}^{(k)}+k \leqslant T_{x^{t}} \leqslant \mathcal{T}_{\ln x-\beta_{1}}^{(k)}+k .
$$

В частности, соотношение (12) справедливо при всех $t \in\left[t_{x}, 1\right]$, где $t_{x}=$ $\ln \left(\sup _{i \leqslant k} \xi_{i}\right) / \ln x$, если при некотором фиксированном $\alpha \in(0,1]$ произошло случайное событие

$$
D_{x, k}^{(\alpha)}:=D_{x, k} \cap\left\{\frac{\ln \left(\sup _{i \leqslant k} \xi_{i}\right)}{\ln x}<\alpha, 2 \xi_{k}<x\right\}
$$

Очевидно, при $\alpha \in(0,1]$

$$
\lim _{x \rightarrow+\infty} \widetilde{\mathbf{P}}\left(D_{x, k} \triangle D_{x, k}^{(\alpha)}\right)=0 .
$$

Заметим (см. (5)), что

$$
\widetilde{\mathbf{E}} X_{1}^{2}=\mathbf{E}\left(X_{1}^{2} \exp \left(\varkappa X_{1}\right)\right), \quad \widetilde{\mathbf{E}}\left(X_{1}-a\right)^{2}=\sigma^{2} .
$$

Положим $\mathcal{T}_{0}=0$ и $\mathcal{T}_{y}=\mathcal{T}_{y}^{(0)}$ при $y>0$. Построенный по случайному блужданию $\left\{S_{n}, n \in \mathbb{N}_{0}\right\}$ с положительным сносом $a$ процесс $\left\{\mathcal{T}_{y}, y \geqslant 0\right\}$ называется, как известно, процессом восстановления. При этом дисперсия $\sigma^{2}$ одного шага случайного блуждания $\left\{S_{n}\right\}$ конечна и положительна.

Для произвольного $y>0$ введем случайный процесс $Z_{y}$ :

$$
Z_{y}(t)=\frac{\mathcal{T}_{t y}-t y / a}{\sigma a^{-3 / 2} \sqrt{y}}, t \geqslant 0 .
$$

Для процессов восстановления справедлива следующая функциональная предельная теорема (см. теорему 3.1 из [7])): при $y \rightarrow \infty$

$$
\left\{Z_{y}(t), t \in[0,1]\right\} \stackrel{\widetilde{D}}{\rightarrow} B
$$


(символ $\sim$ над $D$ означает, что на исходном вероятностном пространстве вместо меры $\mathbf{P}$ рассматривается мера $\widetilde{\mathbf{P}})$.

Для произвольного $y>0$ введем случайную величину

$$
\chi(y)=S_{\mathcal{T}_{y}}-y,
$$

которая называется перескоком блуждания $\left\{S_{n}, n \in \mathbb{N}_{0}\right\}$ через уровень $y$. Известно, что при $y \rightarrow+\infty$

$$
\chi(y) \stackrel{\widetilde{D}}{\rightarrow} \chi,
$$

где $\chi$ - некоторая положительная случайная величина с абсолютно непрерывным распределением.

В добавление к предельным теоремам (14) и (15) можно сказать (см. теорему 8.34 из [8]), что случайная величина $\chi(y)$ и случайный процесс $\left\{Z_{y}(t), t \in[0,1]\right\}$ асимптотически независимы при $y \rightarrow+\infty$. При этом будем считать, что случайные элементы $B$ и $\chi$ определены на одном и том же вероятностном пространстве с мерой $\widehat{\mathbf{P}}$ и независимы.

Для $x \in D[0,1]$ введем модуль непрерывности

$$
w_{x}(\delta)=\sup _{t, s:|t-s| \leqslant \delta}|x(t)-x(s)|, \delta>0 .
$$

При фиксированном $\delta>0$ отображение $f: x \rightarrow w_{x}(\delta), x \in D[0,1]$, является непрерывным при $x \in C[0,1]$. Следовательно, отображение $f$ непрерывно на почти всех броуновских траекториях. Поэтому из соотношения (14) следует, что при $y \rightarrow+\infty$

$$
w_{Z_{y}}(\delta) \stackrel{\widetilde{D}}{\rightarrow} w_{B}(\delta)
$$

Ввиду непрерывности броуновских траекторий почти наверное

$$
\lim _{\delta \rightarrow 0} w_{B}(\delta)=0
$$

Для произвольных $b \in \mathbb{R}$ и $y>0$ введем случайный процесс $Z_{y}^{(b)}$ :

$$
Z_{y}^{(b)}(t)=\left\{\begin{array}{lr}
0, & 0 \leqslant t y \leqslant b \\
\frac{\mathcal{T}_{t y-b}-t y / a}{\sigma a^{-3 / 2} \sqrt{y}}, & b<t y \leqslant 1+b \\
\frac{\mathcal{T}_{y}-y / a}{\sigma a^{-3 / 2} \sqrt{y}}, & 1+b<t y \leqslant y .
\end{array}\right.
$$

Заметим, что

$$
\sup _{t \in[0,1]}\left|Z_{y}^{(b)}(t)-Z_{y}(t)\right| \leqslant w_{Z_{y}}\left(\frac{|b|}{y}\right) .
$$

Откуда, учитывая соотношения (16)-(17), находим, что при $y \rightarrow+\infty$

$$
\sup _{t \in[0,1]}\left|Z_{y}^{(b)}(t)-Z_{y}(t)\right| \stackrel{\widetilde{\mathbf{P}}}{\rightarrow} 0 .
$$

Ввиду соотношений (14) и (18) при $y \rightarrow+\infty$

$$
\left\{Z_{y}^{(b)}(t), t \in[0,1]\right\} \stackrel{\widetilde{D}}{\rightarrow} B
$$


причем левые части соотношений (15) и (19) асимптотически независимы.

Для произвольного $x>1$ введем случайный процесс $Y_{x}$ :

$$
Y_{x}(t)=\frac{T_{x^{t}}-t \ln x / a}{\sigma a^{-3 / 2} \sqrt{\ln x}}, t \geqslant 0
$$

и случайные величины

$$
\varphi_{x}=\frac{\xi_{T_{x}}}{x}, \quad \psi_{x}=\frac{\xi_{T_{x}}}{S_{T_{x}}} .
$$

Заметим, что $\varphi_{x}>1$, если произошло событие $D_{x}$. Введем случайную величину $\widehat{W}$, распределенную так же, как $\{W \mid W>0\}$. Будем считать, что случайная величина $\widehat{W}$ определена на том же вероятностном пространстве, что $B$ и $\chi$, и все эти три случайных элемента независимы.

Покажем, что при $x \rightarrow+\infty$

$$
\begin{gathered}
\left\{Y_{x}(t), t \in[0,1] \mid D_{x}\right\} \stackrel{\widetilde{D}}{\rightarrow} B, \\
\left\{\varphi_{x} \mid D_{x}\right\} \stackrel{\widetilde{D}}{\rightarrow} e^{\chi}, \\
\left\{\psi_{x} \mid D_{x}\right\} \stackrel{\widetilde{D}}{\rightarrow} \widehat{W},
\end{gathered}
$$

причем левые части соотношений (20)-(22) асимптотически независимы.

Сначала установим (20). Введем для произвольных $k \in \mathbb{N}$ и $b \in \mathbb{R}$ случайный процесс $Z_{y}^{(k, b)}$ :

$$
Z_{y}^{(k, b)}(t)=\left\{\begin{array}{cr}
\frac{k}{\sigma a^{-3 / 2} \sqrt{y}}, & 0 \leqslant t y \leqslant b ; \\
\frac{\mathcal{T}_{t y-b}^{(k)}-t y / a+k}{\sigma a^{-3 / 2} \sqrt{y}}, & b<t y \leqslant 1+b ; \\
\frac{\mathcal{T}_{y}^{(k)}-y / a+k}{\sigma a^{-3 / 2} \sqrt{y}}, & 1+b<t y \leqslant y .
\end{array}\right.
$$

Из соотношения (12) вытекает, что если событие $D_{x, k}^{(\alpha)}$ произошло, то при $t \in\left[t_{x}, 1\right]$, где $t_{x}=\ln \left(\sup _{i \leqslant k} \xi_{i}\right) / \ln x<\alpha$,

$$
Z_{y}^{\left(k, \beta_{2}\right)}(t) \leqslant Y_{x}(t) \leqslant Z_{y}^{\left(k, \beta_{1}\right)}(t)
$$

где $y=\ln x$. В силу соотношения (23) для произвольных моментов времени $0<t_{1}<$ $t_{2}<\ldots<t_{m} \leqslant 1$ и чисел $x_{1}, x_{2}, \ldots, x_{m}$, где $m \in \mathbb{N}$,

$$
\begin{gathered}
\widetilde{\mathbf{P}}\left(Z_{y}^{\left(k, \beta_{1}\right)}\left(t_{1}\right) \leqslant x_{1}, \ldots, Z_{y}^{\left(k, \beta_{1}\right)}\left(t_{m}\right) \leqslant x_{m}, D_{x, k}^{\left(t_{1}\right)}\right) \leqslant \\
\leqslant \widetilde{\mathbf{P}}\left(Y_{x}\left(t_{1}\right) \leqslant x_{1}, \ldots, Y_{x}\left(t_{m}\right) \leqslant x_{m}, D_{x, k}^{\left(t_{1}\right)}\right) \leqslant \\
\leqslant \widetilde{\mathbf{P}}\left(Z_{y}^{\left(k, \beta_{2}\right)}\left(t_{1}\right) \leqslant x_{1}, \ldots, Z_{y}^{\left(k, \beta_{2}\right)}\left(t_{m}\right) \leqslant x_{m}, D_{x, k}^{\left(t_{1}\right)}\right) .
\end{gathered}
$$

Откуда, учитывая, что $D_{x, k}^{\left(t_{1}\right)} \subset G_{k}$, находим, что

$$
\begin{gathered}
\widetilde{\mathbf{P}}\left(Z_{y}^{\left(k, \beta_{1}\right)}\left(t_{1}\right) \leqslant x_{1}, \ldots, Z_{y}^{\left(k, \beta_{1}\right)}\left(t_{m}\right) \leqslant x_{m}, G_{k}\right)-\widetilde{\mathbf{P}}\left(G_{k} \triangle D_{x, k}^{\left(t_{1}\right)}\right) \leqslant \\
\leqslant \widetilde{\mathbf{P}}\left(Y_{x}\left(t_{1}\right) \leqslant x_{1}, \ldots, Y_{x}\left(t_{m}\right) \leqslant x_{m}, D_{x, k}^{\left(t_{1}\right)}\right) \leqslant
\end{gathered}
$$




$$
\leqslant \widetilde{\mathbf{P}}\left(Z_{y}^{\left(k, \beta_{2}\right)}\left(t_{1}\right) \leqslant x_{1}, \ldots, Z_{y}^{\left(k, \beta_{2}\right)}\left(t_{m}\right) \leqslant x_{m}, G_{k}\right) .
$$

Покажем, что при $i \in\{1,2\}$

$$
\begin{gathered}
\lim _{y \rightarrow+\infty} \widetilde{\mathbf{P}}\left(Z_{y}^{\left(k, \beta_{i}\right)}\left(t_{1}\right) \leqslant x_{1}, \ldots, Z_{y}^{\left(k, \beta_{i}\right)}\left(t_{m}\right) \leqslant x_{m}, G_{k}\right)= \\
=\widehat{\mathbf{P}}\left(B\left(t_{1}\right) \leqslant x_{1}, \ldots, B\left(t_{m}\right) \leqslant x_{m}\right) \widetilde{\mathbf{P}}\left(G_{k}\right) .
\end{gathered}
$$

Обозначим $\widetilde{\mathbf{P}}_{k}$ и $\widetilde{\mathbf{E}}_{k}$ условную вероятность и условное математическое ожидание относительно $\sigma$-алгебры $\sigma_{k}:=\sigma\left(Q_{1}, \ldots, Q_{k}, \xi_{1}, \ldots, \xi_{k}\right)$ (при этом на исходном вероятностном пространстве вместо меры $\mathbf{P}$ рассматривается мера $\widetilde{\mathbf{P}})$. Тогда, учитывая независимость процесса $\left\{Z_{y}^{(k, b)}(t), t \in[0,1]\right\}$ при фиксированном $b$ от $\sigma_{k}$, видим, что

$$
\begin{gathered}
\widetilde{\mathbf{P}}\left(Z_{y}^{\left(k, \beta_{i}\right)}\left(t_{1}\right) \leqslant x_{1}, \ldots, Z_{y}^{\left(k, \beta_{i}\right)}\left(t_{m}\right) \leqslant x_{m}, G_{k}\right)= \\
=\widetilde{\mathbf{E}}\left(\widetilde{\mathbf{P}}_{k}\left(Z_{y}^{\left(k, \beta_{i}\right)}\left(t_{1}\right) \leqslant x_{1}, \ldots, Z_{y}^{\left(k, \beta_{i}\right)}\left(t_{m}\right) \leqslant x_{m}\right) ; G_{k}\right)= \\
=\widetilde{\mathbf{E}}\left(\left.\widetilde{\mathbf{P}}_{k}\left(Z_{y}^{\left(k, b_{i}\right)}\left(t_{1}\right) \leqslant x_{1}, \ldots, Z_{y}^{\left(k, b_{i}\right)}\left(t_{m}\right) \leqslant x_{m}\right)\right|_{b_{i}=\beta_{i}} ; G_{k}\right)= \\
=\widetilde{\mathbf{E}}\left(\left.\widetilde{\mathbf{P}}\left(Z_{y}^{\left(b_{i}\right)}\left(t_{1}\right)+\frac{k a^{3 / 2}}{\sigma \sqrt{y}} \leqslant x_{1}, \ldots, Z_{y}^{\left(b_{i}\right)}\left(t_{m}\right)+\frac{k a^{3 / 2}}{\sigma \sqrt{y}} \leqslant x_{m}\right)\right|_{b_{i}=\beta_{i}} ; G_{k}\right)
\end{gathered}
$$

(каждая из двух последних вероятностей вычисляется при фиксированном $b_{i}$, a затем в полученное выражение вместо $b_{i}$ подставляется $\beta_{i}$ ). Применяя к этому соотношению утверждение (19), на основании теоремы о мажорируемой сходимости получаем (25).

Теперь перейдем к пределу в соотношении (24) сначала при $x \rightarrow+\infty$, а затем при $k \rightarrow \infty$. Отметим (см. (7) и (8)), что

$$
\begin{aligned}
\lim _{x \rightarrow+\infty} \widetilde{\mathbf{P}}\left(D_{x}\right) & =\widetilde{\mathbf{P}}(D), \\
\lim _{k \rightarrow \infty} \mathbf{P}\left(G_{k}\right) & =\mathbf{P}(D) .
\end{aligned}
$$

Из соотношений $(10),(25)$ и (27) находим, что

$$
\begin{gathered}
\lim _{k \rightarrow \infty} \lim _{x \rightarrow+\infty} \widetilde{\mathbf{P}}\left(Y_{x}\left(t_{1}\right) \leqslant x_{1}, \ldots, Y_{x}\left(t_{m}\right) \leqslant x_{m}, D_{x, k}^{\left(t_{1}\right)}\right)= \\
=\widehat{\mathbf{P}}\left(B\left(t_{1}\right) \leqslant x_{1}, \ldots, B\left(t_{m}\right) \leqslant x_{m}\right) \widetilde{\mathbf{P}}(D) .
\end{gathered}
$$

Поскольку $D_{x, k}^{\left(t_{1}\right)} \subset D_{x, k} \subset D_{x}$, то

$$
\begin{gathered}
\widetilde{\mathbf{P}}\left(Y_{x}\left(t_{1}\right) \leqslant x_{1}, \ldots, Y_{x}\left(t_{m}\right) \leqslant x_{m}, D_{x, k}^{\left(t_{1}\right)}\right) \leqslant \\
\leqslant \widetilde{\mathbf{P}}\left(Y_{x}\left(t_{1}\right) \leqslant x_{1}, \ldots, Y_{x}\left(t_{m}\right) \leqslant x_{m}, D_{x}\right) \leqslant \\
\leqslant \widetilde{\mathbf{P}}\left(Y_{x}\left(t_{1}\right) \leqslant x_{1}, \ldots, Y_{x}\left(t_{m}\right) \leqslant x_{m}, D_{x, k}^{\left(t_{1}\right)}\right)+ \\
\quad+\widetilde{\mathbf{P}}\left(D_{x} \triangle D_{x, k}\right)+\widetilde{\mathbf{P}}\left(D_{x, k} \triangle D_{x, k}^{\left(t_{1}\right)}\right) .
\end{gathered}
$$


Откуда, переходя к пределу при $x \rightarrow+\infty$, а затем при $k \rightarrow \infty$ и применяя соотношения (9), (13) и (28), видим, что

$$
\begin{gathered}
\lim _{x \rightarrow+\infty} \widetilde{\mathbf{P}}\left(Y_{x}\left(t_{1}\right) \leqslant x_{1}, \ldots, Y_{x}\left(t_{m}\right) \leqslant x_{m}, D_{x}\right)= \\
=\widehat{\mathbf{P}}\left(B\left(t_{1}\right) \leqslant x_{1}, \ldots, B\left(t_{m}\right) \leqslant x_{m}\right) \widetilde{\mathbf{P}}(D) .
\end{gathered}
$$

Учитывая теперь (26), получаем, что

$$
\begin{gathered}
\lim _{x \rightarrow+\infty} \widetilde{\mathbf{P}}\left(Y_{x}\left(t_{1}\right) \leqslant x_{1}, \ldots, Y_{x}\left(t_{m}\right) \leqslant x_{m} \mid D_{x}\right)= \\
=\widehat{\mathbf{P}}\left(B\left(t_{1}\right) \leqslant x_{1}, \ldots, B\left(t_{m}\right) \leqslant x_{m}\right) .
\end{gathered}
$$

Итак, сходимость конечномерных распределений в (20) установлена.

Покажем, что для произвольного $\varepsilon^{\prime}>0$

$$
\lim _{\delta \rightarrow 0} \limsup _{k \rightarrow \infty} \limsup _{x \rightarrow+\infty} \widetilde{\mathbf{P}}\left(w_{Y_{x}}(\delta) \geqslant \varepsilon^{\prime} ; D_{x, k}^{(1)}\right)=0 .
$$

Нетрудно заметить, что если $x_{1}, x_{2}, x_{3} \in D[0,1]$ и $x_{1}(t) \leqslant x_{2}(t) \leqslant x_{3}(t)$ при всех $t \in\left[t_{0}, 1\right]$, где $t_{0} \in(0,1)$, то при всех $\delta>0$

$$
w_{x_{2}}(\delta) \leqslant 2 \sup _{t \in\left[0, t_{0}\right)}\left|x_{2}(t)\right|+w_{x_{1}}(\delta)+\sup _{t \in[0,1]}\left|x_{3}(t)-x_{1}(t)\right| .
$$

Откуда с учетом (23) находим, что если событие $D_{x, k}^{(1)}$ произошло, то при $t_{x}=$ $\ln \left(\sup _{i \leqslant k} \xi_{i}\right) / \ln x$

$$
w_{Y_{x}}(\delta) \leqslant 2 \sup _{t \in\left[0, t_{x}\right)}\left|Y_{x}(t)\right|+w_{Z_{y}^{\left(k, \beta_{1}\right)}}(\delta)+\sup _{t \in[0,1]}\left|Z_{y}^{\left(k, \beta_{2}\right)}(t)-Z_{y}^{\left(k, \beta_{1}\right)}(t)\right|,
$$

где $y=\ln x$. Это означает, что для справедливости (29) достаточно показать, что

$$
\begin{gathered}
\lim _{\delta \rightarrow 0} \limsup _{k \rightarrow \infty} \limsup _{x \rightarrow+\infty} \widetilde{\mathbf{P}}\left(2 \sup _{t \in\left[0, t_{x}\right)}\left|Y_{x}(t)\right| \geqslant \frac{\varepsilon^{\prime}}{3}\right)=0, \\
\lim _{\delta \rightarrow 0} \limsup _{k \rightarrow \infty} \limsup _{x \rightarrow+\infty} \widetilde{\mathbf{P}}\left(w_{Z_{y}^{\left(k, \beta_{1}\right)}}(\delta) \geqslant \frac{\varepsilon^{\prime}}{3} ; D_{x, k}\right)=0, \\
\limsup _{k \rightarrow \infty} \limsup _{x \rightarrow+\infty} \widetilde{\mathbf{P}}\left(\sup _{t \in[0,1]}\left|Z_{y}^{\left(k, \beta_{2}\right)}(t)-Z_{y}^{\left(k, \beta_{1}\right)}(t)\right| \geqslant \frac{\varepsilon^{\prime}}{3} ; D_{x, k}\right)=0
\end{gathered}
$$

(здесь учтено, что $D_{x, k}^{(1)} \subset D_{x, k}$ ).

Заметим, что если $t<t_{x}$, то $t \ln x<\ln \left(\sup _{i \leqslant k} \xi_{i}\right)$ и, значит, $T_{x^{t}} \leqslant k$, поэтому

$$
\sup _{t \in\left[0, t_{x}\right)}\left|Y_{x}(t)\right| \leqslant \frac{k+\ln \left(\sup _{i \leqslant k} \xi_{i}\right)}{\sigma a^{-3 / 2} \sqrt{\ln x}}
$$

откуда следует (31).

Теперь установим (32). Поскольку $D_{x, k} \subset G_{k}$, то

$$
\widetilde{\mathbf{P}}\left(w_{Z_{y}^{\left(k, \beta_{1}\right)}}(\delta) \geqslant \frac{\varepsilon^{\prime}}{3} ; D_{x, k}\right) \leqslant \widetilde{\mathbf{P}}\left(w_{Z_{y}^{\left(k, \beta_{1}\right)}}(\delta) \geqslant \frac{\varepsilon^{\prime}}{3} ; G_{k}\right)+\widetilde{\mathbf{P}}\left(G_{k} \backslash D_{x, k}\right) .
$$


Заметим, что

$$
\begin{aligned}
\widetilde{\mathbf{P}}\left(w_{Z_{y}^{\left(k, \beta_{1}\right)}}(\delta) \geqslant \frac{\varepsilon^{\prime}}{3} ; G_{k}\right)=\widetilde{\mathbf{E}}\left(\widetilde{\mathbf{P}}_{k}\left(w_{Z_{y}^{\left(k, \beta_{1}\right)}}(\delta) \geqslant \frac{\varepsilon^{\prime}}{3}\right) ; G_{k}\right)= \\
=\widetilde{\mathbf{E}}\left(\left.\widetilde{\mathbf{P}}_{k}\left(w_{Z_{y}^{\left(k, b_{1}\right)}}(\delta) \geqslant \frac{\varepsilon^{\prime}}{3}\right)\right|_{b_{1}=\beta_{1}} ; G_{k}\right)= \\
=\widetilde{\mathbf{E}}\left(\left.\widetilde{\mathbf{P}}\left(w_{Z_{y}^{\left(b_{1}\right)}}(\delta) \geqslant \frac{\varepsilon^{\prime}}{3}\right)\right|_{b_{1}=\beta_{1}} ; G_{k}\right) .
\end{aligned}
$$

Из соотношения (19) вытекает, что при $y \rightarrow+\infty$

$$
w_{Z_{y}^{\left(b_{1}\right)}}(\delta) \stackrel{\widetilde{D}}{\rightarrow} w_{B}(\delta) .
$$

Из соотношений (35), (36) по теореме о мажорируемой сходимости следует, что

$$
\lim _{y \rightarrow+\infty} \widetilde{\mathbf{P}}\left(w_{Z_{y}^{\left(k, \beta_{1}\right)}}(\delta) \geqslant \frac{\varepsilon^{\prime}}{3} ; G_{k}\right)=\widehat{\mathbf{P}}\left(w_{B}(\delta) \geqslant \frac{\varepsilon^{\prime}}{3}\right) \widetilde{\mathbf{P}}\left(G_{k}\right)
$$

при всех $\varepsilon^{\prime}>0$ за исключением не более чем счетного множества. Откуда, учитывая (17), находим, что

$$
\lim _{\delta \rightarrow 0} \limsup _{k \rightarrow \infty} \limsup _{y \rightarrow+\infty} \widetilde{\mathbf{P}}\left(w_{Z_{y}^{\left(k, \beta_{1}\right)}}(\delta) \geqslant \frac{\varepsilon^{\prime}}{3} ; G_{k}\right)=0 .
$$

Переходя в (34) к пределу при $x \rightarrow+\infty$, а затем при $k \rightarrow \infty$ и применяя соотношения (10) и (37), получаем (32).

Наконец, установим (33). Поскольку $D_{x, k} \subset G_{k}$, то

$$
\begin{gathered}
\widetilde{\mathbf{P}}\left(\sup _{t \in[0,1]}\left|Z_{y}^{\left(k, \beta_{2}\right)}(t)-Z_{y}^{\left(k, \beta_{1}\right)}(t)\right| \geqslant \frac{\varepsilon^{\prime}}{3} ; D_{x, k}\right) \leqslant \\
\leqslant \widetilde{\mathbf{P}}\left(\sup _{t \in[0,1]}\left|Z_{y}^{\left(k, \beta_{2}\right)}(t)-Z_{y}^{\left(k, \beta_{1}\right)}(t)\right| \geqslant \frac{\varepsilon^{\prime}}{3} ; G_{k}\right)+\widetilde{\mathbf{P}}\left(G_{k} \backslash D_{x, k}\right) .
\end{gathered}
$$

Очевидно, что

$$
\begin{gathered}
\widetilde{\mathbf{P}}\left(\sup _{t \in[0,1]}\left|Z_{y}^{\left(k, \beta_{2}\right)}(t)-Z_{y}^{\left(k, \beta_{1}\right)}(t)\right| \geqslant \frac{\varepsilon^{\prime}}{3} ; G_{k}\right)= \\
=\widetilde{\mathbf{E}}\left(\widetilde{\mathbf{P}}_{k}\left(\sup _{t \in[0,1]}\left|Z_{y}^{\left(k, \beta_{2}\right)}(t)-Z_{y}^{\left(k, \beta_{1}\right)}(t)\right| \geqslant \frac{\varepsilon^{\prime}}{3}\right) ; G_{k}\right)= \\
=\widetilde{\mathbf{E}}\left(\left.\widetilde{\mathbf{P}}_{k}\left(\sup _{t \in[0,1]}\left|Z_{y}^{\left(k, b_{2}\right)}(t)-Z_{y}^{\left(k, b_{1}\right)}(t)\right| \geqslant \frac{\varepsilon^{\prime}}{3}\right)\right|_{b_{1}=\beta_{1}, b_{2}=\beta_{2}} ; G_{k}\right)= \\
=\widetilde{\mathbf{E}}\left(\left.\widetilde{\mathbf{P}}\left(\sup _{t \in[0,1]}\left|Z_{y}^{\left(b_{2}\right)}(t)-Z_{y}^{\left(b_{1}\right)}(t)\right| \geqslant \frac{\varepsilon^{\prime}}{3}\right)\right|_{b_{1}=\beta_{1}, b_{2}=\beta_{2}} ; G_{k}\right) .
\end{gathered}
$$


Применяя к последнему соотношению утверждение (18), находим по теореме о мажорируемой сходимости

$$
\lim _{y \rightarrow+\infty} \widetilde{\mathbf{P}}\left(\sup _{t \in[0,1]}\left|Z_{y}^{\left(k, \beta_{2}\right)}(t)-Z_{y}^{\left(k, \beta_{1}\right)}(t)\right| \geqslant \frac{\varepsilon^{\prime}}{3} ; G_{k}\right)=0 .
$$

Переходя в (38) к пределу при $x \rightarrow+\infty$, а затем при $k \rightarrow \infty$ и применяя соотношения (10) и (39), получаем (33).

Итак, соотношение (29) доказано. Поскольку $D_{x, k}^{(1)} \subset D_{x, k} \subset D_{x}$, то

$$
\begin{gathered}
\widetilde{\mathbf{P}}\left(w_{Y_{x}}(\delta) \geqslant \varepsilon^{\prime} ; D_{x}\right) \leqslant \\
\leqslant \widetilde{\mathbf{P}}\left(w_{Y_{x}}(\delta) \geqslant \varepsilon^{\prime} ; D_{x, k}^{(1)}\right)+\widetilde{\mathbf{P}}\left(D_{x} \triangle D_{x, k}\right)+\widetilde{\mathbf{P}}\left(D_{x, k} \triangle D_{x, k}^{(1)}\right) .
\end{gathered}
$$

Откуда, переходя к пределу при $x \rightarrow+\infty$, а затем при $k \rightarrow \infty$ и учитывая (9), (13), (29) и (26), получаем, что

$$
\lim _{\delta \rightarrow 0} \limsup _{x \rightarrow+\infty} \widetilde{\mathbf{P}}\left(w_{Y_{x}}(\delta) \geqslant \varepsilon^{\prime} \mid D_{x}\right)=0 .
$$

Это утверждение вместе со сходимостью конечномерных распределений означает справедливость соотношения (20).

Перейдем к доказательству (21) и (22), объединив их в одно соотношение:

$$
\left\{\varphi_{x}, \psi_{x} \mid D_{x}\right\} \stackrel{\widetilde{D}}{\rightarrow}\left(e^{\chi}, \widehat{W}\right) .
$$

Заметим, что при $u>1, z>0$ и $l>k$

$$
\begin{gathered}
\left\{\varphi_{x}>u, \psi_{x} \leqslant z, T_{x}=l, D_{x, k}\right\}= \\
=\left\{\xi_{k+1} \leqslant x, \ldots, \xi_{l-1} \leqslant x, \xi_{l}>x u, \frac{\xi_{l}}{e^{S_{l}}} \leqslant z, D_{x, k}\right\}= \\
=\left\{e^{S_{k+1}} \leqslant x \frac{e^{S_{k+1}}}{\xi_{k+1}}, \ldots, e^{S_{l-1}} \leqslant x \frac{e^{S_{l-1}}}{\xi_{l-1}}, e^{S_{l}}>x u \frac{e^{S_{l}}}{\xi_{l}}, \frac{e^{S_{l}}}{\xi_{l}} \geqslant \frac{1}{z}, D_{x, k}\right\} .
\end{gathered}
$$

Положим

$$
L=\frac{(1-\varepsilon) x}{\xi_{k}}, \quad M=\frac{(1+\varepsilon) x}{\xi_{k}} .
$$

Событие $(41)$ при $u>(1+\varepsilon) /(1-\varepsilon)$ влечет (см. определение $\left.D_{x, k}\right)$ событие

$$
\begin{gathered}
\left\{e^{S_{k+1}} \leqslant M e^{S_{k}}, \ldots, e^{S_{l-1}} \leqslant M e^{S_{k}}, e^{S_{l}}>u L e^{S_{k}}, M e^{S_{k}} \geqslant \frac{x}{z}, D_{x, k}\right\}= \\
=\left\{S_{1}^{(k)} \leqslant \ln M, \ldots, S_{l-1-k}^{(k)} \leqslant \ln M, S_{l-k}^{(k)}>\ln (u L), M e^{S_{k}} \geqslant \frac{x}{z}, D_{x, k}\right\}= \\
=\left\{\chi^{(k)}\left(y-\beta_{1}\right)>v+\ln \frac{1-\varepsilon}{1+\varepsilon}, \mathcal{T}_{y-\beta_{1}}^{(k)}=l-k, M e^{S_{k}} \geqslant \frac{x}{z}, D_{x, k}\right\},
\end{gathered}
$$

где $v=\ln u, y=\ln x$, а $\chi^{(k)}(y)$ - перескок блуждания $\left\{S_{n}^{(k)}, n \in \mathbb{N}_{0}\right\}$ через уровень $y>0$. Следовательно,

$$
\left\{\varphi_{x}>u, \psi_{x} \leqslant z, D_{x, k}\right\} \subset\left\{\chi^{(k)}\left(y-\beta_{1}\right)>v+\ln \frac{1-\varepsilon}{1+\varepsilon}, M e^{S_{k}} \geqslant \frac{x}{z}, D_{x, k}\right\} .
$$


С другой стороны, событие (41) вытекает из события

$$
\begin{gathered}
\left\{e^{S_{k+1}} \leqslant L e^{S_{k}}, \ldots, e^{S_{l-1}} \leqslant L e^{S_{k}}, e^{S_{l}}>u M e^{S_{k}}, L e^{S_{k}} \geqslant \frac{x}{z}, D_{x, k}\right\}= \\
=\left\{S_{1}^{(k)} \leqslant \ln L, \ldots, S_{l-1-k}^{(k)} \leqslant \ln L, S_{l-k}^{(k)}>\ln (u M), L e^{S_{k}} \geqslant \frac{x}{z}, D_{x, k}\right\}= \\
=\left\{\chi^{(k)}\left(y-\beta_{2}\right)>v+\ln \frac{1+\varepsilon}{1-\varepsilon}, \mathcal{T}_{y-\beta_{2}}^{(k)}=l-k, L e^{S_{k}} \geqslant \frac{x}{z}, D_{x, k}\right\} .
\end{gathered}
$$

Следовательно,

$$
\left\{\varphi_{x}>u, \psi_{x} \leqslant z, D_{x, k}\right\} \supset\left\{\chi^{(k)}\left(y-\beta_{2}\right)>v+\ln \frac{1+\varepsilon}{1-\varepsilon}, L e^{S_{k}} \geqslant \frac{x}{z}, D_{x, k}\right\} .
$$

Из соотношений (42), (43) видно, что

$$
\begin{gathered}
\widetilde{\mathbf{P}}\left(\chi^{(k)}\left(y-\beta_{2}\right)>v+\ln \frac{1+\varepsilon}{1-\varepsilon}, L e^{S_{k}} \geqslant \frac{x}{z}, D_{x, k}\right) \\
\leqslant \widetilde{\mathbf{P}}\left(\varphi_{x}>u, \psi_{x} \leqslant z, D_{x, k}\right) \leqslant \\
\leqslant \widetilde{\mathbf{P}}\left(\chi^{(k)}\left(y-\beta_{1}\right)>v+\ln \frac{1-\varepsilon}{1+\varepsilon}, M e^{S_{k}} \geqslant \frac{x}{z}, D_{x, k}\right) .
\end{gathered}
$$

Поскольку $D_{x, k} \subset G_{k}$, из соотношения (44) находим, что

$$
\begin{gathered}
\widetilde{\mathbf{P}}\left(\chi^{(k)}\left(y-\beta_{2}\right)>\right. \\
\left.v+\ln \frac{1+\varepsilon}{1-\varepsilon}, L e^{S_{k}} \geqslant \frac{x}{z}, G_{k}\right)-\widetilde{\mathbf{P}}\left(G_{k} \backslash D_{x, k}\right) \leqslant \\
\leqslant \widetilde{\mathbf{P}}\left(\varphi_{x}>u, \psi_{x} \leqslant z, D_{x, k}\right) \leqslant \\
\leqslant \widetilde{\mathbf{P}}\left(\chi^{(k)}\left(y-\beta_{1}\right)>v+\ln \frac{1-\varepsilon}{1+\varepsilon}, M e^{S_{k}} \geqslant \frac{x}{z}, G_{k}\right) .
\end{gathered}
$$

Заметим, что

$$
\begin{aligned}
& \widetilde{\mathbf{P}}\left(\chi^{(k)}\left(y-\beta_{2}\right)>v+\ln \frac{1+\varepsilon}{1-\varepsilon}, L e^{S_{k}} \geqslant \frac{x}{z}, G_{k}\right)= \\
= & \widetilde{\mathbf{E}}\left(\widetilde{\mathbf{P}}_{k}\left(\chi^{(k)}\left(y-\beta_{2}\right)>v+\ln \frac{1+\varepsilon}{1-\varepsilon}\right) ; L e^{S_{k}} \geqslant \frac{x}{z}, G_{k}\right)= \\
= & \widetilde{\mathbf{E}}\left(\left.\widetilde{\mathbf{P}}_{k}\left(\chi^{(k)}\left(y-b_{2}\right)>v+\ln \frac{1+\varepsilon}{1-\varepsilon}\right)\right|_{b_{2}=\beta_{2}} ; L e^{S_{k}} \geqslant \frac{x}{z}, G_{k}\right)= \\
= & \widetilde{\mathbf{E}}\left(\left.\widetilde{\mathbf{P}}\left(\chi\left(y-b_{2}\right)>v+\ln \frac{1+\varepsilon}{1-\varepsilon}\right)\right|_{b_{2}=\beta_{2}} ; L e^{S_{k}} \geqslant \frac{x}{z}, G_{k}\right) .
\end{aligned}
$$

Откуда, учитывая (15), получаем на основании теоремы о мажорируемой сходимости, что

$$
\begin{gathered}
\lim _{y \rightarrow+\infty} \widetilde{\mathbf{P}}\left(\chi^{(k)}\left(y-\beta_{2}\right)>v+\ln \frac{1+\varepsilon}{1-\varepsilon}, L e^{S_{k}} \geqslant \frac{x}{z}, G_{k}\right)= \\
=\widehat{\mathbf{P}}\left(\chi>v+\ln \frac{1+\varepsilon}{1-\varepsilon}\right) \widetilde{\mathbf{P}}\left(L e^{S_{k}} \geqslant \frac{x}{z}, G_{k}\right) .
\end{gathered}
$$


Аналогично устанавливается, что

$$
\begin{aligned}
\lim _{y \rightarrow+\infty} & \widetilde{\mathbf{P}}\left(\chi^{(k)}\left(y-\beta_{1}\right)>v+\ln \frac{1-\varepsilon}{1+\varepsilon}, M e^{S_{k}} \geqslant \frac{x}{z}, G_{k}\right)= \\
= & \widehat{\mathbf{P}}\left(\chi>v+\ln \frac{1-\varepsilon}{1+\varepsilon}\right) \widetilde{\mathbf{P}}\left(M e^{S_{k}} \geqslant \frac{x}{z}, G_{k}\right) .
\end{aligned}
$$

Далее, в силу соотношений (6) и (8)

$$
\begin{gathered}
\lim _{k \rightarrow \infty} \widetilde{\mathbf{P}}\left(L e^{S_{k}} \geqslant \frac{x}{z}, G_{k}\right)=\lim _{k \rightarrow \infty} \widetilde{\mathbf{P}}\left(\frac{\xi_{k}}{\exp S_{k}} \leqslant(1-\varepsilon) z, G_{k}\right)= \\
=\widetilde{\mathbf{P}}(W \leqslant(1-\varepsilon) z, D), \\
\lim _{k \rightarrow \infty} \widetilde{\mathbf{P}}\left(M e^{S_{k}} \geqslant \frac{x}{z}, G_{k}\right)=\lim _{k \rightarrow \infty} \widetilde{\mathbf{P}}\left(\frac{\xi_{k}}{\exp S_{k}} \leqslant(1+\varepsilon) z, G_{k}\right)= \\
=\widetilde{\mathbf{P}}(W \leqslant(1+\varepsilon) z, D)
\end{gathered}
$$

для всех $\varepsilon \in(0,1)$ за исключением не более чем счетного множества. Перейдем в соотношении (45) к пределу сначала при $x \rightarrow+\infty$, а затем при $k \rightarrow \infty$. Учитывая соотношения (10), (47)-(49), получаем, что

$$
\begin{gathered}
\widehat{\mathbf{P}}\left(\chi>v+\ln \frac{1+\varepsilon}{1-\varepsilon}\right) \widetilde{\mathbf{P}}(W \leqslant(1-\varepsilon) z, D) \leqslant \\
\leqslant \liminf _{x \rightarrow+\infty} \widetilde{\mathbf{P}}\left(\varphi_{x}>u, \psi_{x} \leqslant z, D_{x}\right) \leqslant \\
\leqslant \limsup _{x \rightarrow+\infty} \widetilde{\mathbf{P}}\left(\varphi_{x}>u, \psi_{x} \leqslant z, D_{x}\right) \leqslant \\
\leqslant \widehat{\mathbf{P}}\left(\chi>v+\ln \frac{1-\varepsilon}{1+\varepsilon}\right) \widetilde{\mathbf{P}}(W \leqslant(1+\varepsilon) z, D),
\end{gathered}
$$

Наконец, переходя в (50) к пределу при $\varepsilon \rightarrow 0$, находим, что

$$
\begin{gathered}
\lim _{x \rightarrow+\infty} \widetilde{\mathbf{P}}\left(\varphi_{x}>u, \psi_{x}>z, D_{x}\right)=\widehat{\mathbf{P}}(\chi>v) \widetilde{\mathbf{P}}(W \leqslant z, D)= \\
=\widehat{\mathbf{P}}\left(e^{\chi}>u\right) \widetilde{\mathbf{P}}(W \leqslant z, D),
\end{gathered}
$$

если $z$ является точкой непрерывности функции распределения случайной величины $W$. Откуда, заменяя $D$ на $D^{*}$ и учитывая (26), получаем (40).

Объединяя левые части соотношений (20) и (40) и повторяя рассуждения, использованные при их доказательстве, а также используя асимптотическую независимость случайных элементов $\left\{Z_{y}^{(b)}(t), t \in[0,1]\right\}$ и $\chi(y)$ при $y \rightarrow+\infty$, нетрудно доказать асимптотическую независимость левых частей соотношений (20) и (40) при $x \rightarrow+\infty$ и, следовательно, асимптотическую независимость левых частей соотношений (20)-(22).

Завершим доказательство теоремы. Пусть $f$ - произвольная ограниченная непрерывная числовая функция, заданная на $D[0,1]$. Заметим, что

$$
\mathbf{E}\left(f\left(Y_{x}\right) ; D_{x}\right)=\widetilde{\mathbf{E}}\left(\exp \left(-\varkappa S_{T_{x}}\right) f\left(Y_{x}\right) ; D_{x}\right) .
$$


Действительно,

$$
\mathbf{E}\left(f\left(Y_{x}\right) ; D_{x}\right)=\sum_{m=1}^{\infty} \mathbf{E}\left(f\left(Y_{x}\right) ; T_{x}=m\right) .
$$

Случайная величина $f\left(Y_{x}\right) I\left(T_{x}=m\right)$ измерима относительно $\sigma$-алгебры $\sigma_{m}$ и, следовательно (см. (5)),

$$
\begin{gathered}
\mathbf{E}\left(f\left(Y_{x}\right) ; T_{x}=m\right)=\widetilde{\mathbf{E}}\left(\exp \left(-\varkappa S_{m}\right) f\left(Y_{x}\right) ; T_{x}=m\right)= \\
=\widetilde{\mathbf{E}}\left(\exp \left(-\varkappa S_{T_{x}}\right) f\left(Y_{x}\right) ; T_{x}=m\right) .
\end{gathered}
$$

Таким образом,

$$
\begin{aligned}
\mathbf{E}\left(f\left(Y_{x}\right) ; D_{x}\right) & =\sum_{m=1}^{\infty} \widetilde{\mathbf{E}}\left(\exp \left(-\varkappa S_{T_{x}}\right) f\left(Y_{x}\right) ; T_{x}=m\right)= \\
& =\widetilde{\mathbf{E}}\left(\exp \left(-\varkappa S_{T_{x}}\right) f\left(Y_{x}\right)\right) .
\end{aligned}
$$

Итак, соотношение (51) доказано.

Перепишем соотношение (51) в виде

$$
\mathbf{E}\left(f\left(Y_{x}\right) ; D_{x}\right)=x^{-\varkappa} \widetilde{\mathbf{E}}\left(\left(\frac{\psi_{x}}{\varphi_{x}}\right)^{\varkappa} f\left(Y_{x}\right) ; D_{x}\right) .
$$

При $f \equiv 1$ находим, что

$$
\mathbf{P}\left(D_{x}\right)=x^{-\varkappa} \widetilde{\mathbf{E}}\left(\left(\frac{\psi_{x}}{\varphi_{x}}\right)^{\varkappa} ; D_{x}\right) .
$$

Разделив (52) на (53), получаем, что

$$
\mathbf{E}\left(f\left(Y_{x}\right) \mid D_{x}\right)=\widetilde{\mathbf{E}}\left(\left(\frac{\psi_{x}}{\varphi_{x}}\right)^{\varkappa} f\left(Y_{x}\right) \mid D_{x}\right)\left(\widetilde{\mathbf{E}}\left(\left(\frac{\psi_{x}}{\varphi_{x}}\right)^{\varkappa} \mid D_{x}\right)\right)^{-1} .
$$

Из соотношений (20)-(22) следует, что

$$
\left\{\left(\frac{\psi_{x}}{\varphi_{x}}\right)^{\varkappa} f\left(Y_{x}\right) \mid D_{x}\right\} \stackrel{\widetilde{D}}{\rightarrow}(\widehat{W})^{\varkappa} e^{-\varkappa \chi} f(B) .
$$

Далее,

$$
\left(\frac{\psi_{x}}{\varphi_{x}}\right)^{\varkappa} f\left(Y_{x}\right) \leqslant K\left(\psi_{x}\right)^{\varkappa},
$$

где $K>0$ - постоянная, ограничивающая модуль функции $f$. Поскольку семейство случайных величин $\left(\psi_{x}\right)^{\varkappa}$, где $x>1$, равномерно интегрируемо, то равномерно интегрируемо семейство $\left\{\left(\psi_{x} / \varphi_{x}\right)^{\varkappa} f\left(Y_{x}\right), x>1\right\}$ и, следовательно (см. (26)), семейство случайных величин $\left\{\left(\psi_{x} / \varphi_{x}\right)^{\varkappa} f\left(Y_{x}\right) \mid D_{x}\right\}$, где $x>1$. В силу сказанного (см. теорему 5.4 из [9])

$$
\begin{gathered}
\lim _{x \rightarrow+\infty} \widetilde{\mathbf{E}}\left(\left(\frac{\psi_{x}}{\varphi_{x}}\right)^{\varkappa}\right. \\
\left.f\left(Y_{x}\right) \mid D_{x}\right)=\widehat{\mathbf{E}}\left((\widehat{W})^{\varkappa} e^{-\varkappa \chi} f(B)\right)= \\
=\widehat{\mathbf{E}}(\widehat{W})^{\varkappa} \widehat{\mathbf{E}} e^{-\varkappa \chi} \widehat{\mathbf{E}} f(B)
\end{gathered}
$$


и (при $f \equiv 1)$

$$
\lim _{x \rightarrow+\infty} \widetilde{\mathbf{E}}\left(\left(\frac{\psi_{x}}{\varphi_{x}}\right)^{\varkappa} \mid D_{x}\right)=\widehat{\mathbf{E}}(\widehat{W})^{\varkappa} \widehat{\mathbf{E}} e^{-\varkappa \chi} .
$$

Из соотношений (54)-(56) получаем, что

$$
\lim _{x \rightarrow+\infty} \mathbf{E}\left(f\left(Y_{x}\right) \mid D_{x}\right)=\widehat{\mathbf{E}} f(B) .
$$

Теорема 1 доказана.

Замечание 1. Из соотношений $(53),(56)$ и (26) следует, что при $x \rightarrow \infty$

$$
\mathbf{P}\left(D_{x}\right) \sim c_{0} x^{-\varkappa}
$$

где

$$
c_{0}=\widehat{\mathbf{E}}(\widehat{W})^{\varkappa} \widehat{\mathbf{E}} e^{-\varkappa \chi} \widetilde{\mathbf{P}}(D)=\widetilde{\mathbf{E}} W^{\varkappa} \widehat{\mathbf{E}} e^{-\varkappa \chi},
$$

тем самым, асимптотика $\mathbf{P}\left(D_{x}\right)$ найдена несколько иным по сравнению с [2] способом.

4. Приступим к доказательству теоремъ 2. Для произвольного $y>0$ введем случайный процесс $U_{y}$ :

$$
U_{y}(t)=\frac{\ln \xi_{\lfloor t y / a\rfloor}-t y}{\sigma \sqrt{y / a}}, t \geqslant 0,
$$

где для определенности полагаем $\ln 0=-\infty$. Для произвольных $k \in \mathbb{N}_{0}, b \in \mathbb{R}$ и $y>0$ введем случайный процесс $V_{y}^{(k, b)}$ :

$$
V_{y}^{(k, b)}(t)=\frac{S_{(\lfloor t y / a\rfloor-k)^{+}}^{(k)}-t y+b}{\sigma \sqrt{y / a}}, t \geqslant 0 .
$$

Положим $x=\exp (y)$. Из соотношения (11) следует, что если событие $D_{x, k}$ произошло, то при $t \in\left[t_{y}, 1\right]$, где $t_{y}=(k+1) a / y$,

$$
V_{y}^{\left(k, \beta_{1}\right)}(t) \leqslant U_{y}(t) \leqslant V_{y}^{\left(k, \beta_{2}\right)}(t)
$$

(при этом выбираем $y$ так, чтобы $(k+1) a<y)$.

В отличие от доказательства теоремы 1 , опирающегося на предельную теорему (14), доказательство теоремы 2 основывается на следующем варианте принципа инвариантности Донскера-Прохорова для процесса $V_{y}=V_{y}^{(0,0)}:$ при $y \rightarrow \infty$

$$
\left\{V_{y}(t), t \in[0,1]\right\} \stackrel{\widetilde{D}}{\rightarrow} B
$$

причем для произвольного $u \in(0,1)$ процесс $\left\{V_{y}(t), t \in[0, u]\right\}$ и левая часть соотношения (15) асимптотически независимы.

Докажем последнее утверждение. При этом ограничимся рассмотрением одномерного сечения процесса $V_{y}$ в момент времени $t \in(0, u]$. Заметим, что при $z \in \mathbb{R}$

$$
\left\{V_{y}(t) \leqslant z\right\} \supset\left\{\mathcal{T}_{Z}>\lfloor t y / a\rfloor\right\},
$$

где $Z=t y+z \sigma \sqrt{y / a}$. С другой стороны, для произвольного $l>0$

$$
\left\{V_{y}(t) \leqslant z\right\} \subset\left\{\mathcal{T}_{Z+l}>\lfloor t y / a\rfloor\right\} \cup\left\{M_{\lfloor t y / a\rfloor}-S_{\lfloor t y / a\rfloor}>l\right\},
$$


где $M_{n}=\max _{i \leqslant n} S_{i}$ при $n \in \mathbb{N}_{0}$. Следовательно, при $v \in(0,+\infty)$

$$
\begin{aligned}
& \widetilde{\mathbf{P}}\left(\mathcal{T}_{Z}>\lfloor t y / a\rfloor, \chi(y)>v\right) \leqslant \widetilde{\mathbf{P}}\left(V_{y}(t) \leqslant z, \chi(y)>v\right) \leqslant \\
\leqslant & \widetilde{\mathbf{P}}\left(\mathcal{T}_{Z+l}>\lfloor t y / a\rfloor, \chi(y)>v\right)+\widetilde{\mathbf{P}}\left(M_{\lfloor t y / a\rfloor}-S_{\lfloor t y / a\rfloor}>l\right) .
\end{aligned}
$$

Хорошо известно, что при $n \in \mathbb{N}_{0}$

$$
S_{n}-M_{n} \stackrel{d}{=} L_{n}
$$

где $L_{n}=\min _{i \leqslant n} S_{i}$. Поскольку $\widetilde{\mathbf{E}} X_{1}>0$, то при $n \rightarrow \infty$

$$
L_{n} \stackrel{\widetilde{D}}{\rightarrow} L,
$$

где $L$ - некоторая неположительная случайная величина. Следовательно,

$$
\lim _{l \rightarrow+\infty} \limsup _{y \rightarrow+\infty} \widetilde{\mathbf{P}}\left(M_{\lfloor t y / a\rfloor}-S_{\lfloor t y / a\rfloor}>l\right)=0 .
$$

Ввиду соотношения (15) и асимптотической независимости левых частей соотношений (14) и (15)

$$
\begin{gathered}
\lim _{x \rightarrow+\infty} \widetilde{\mathbf{P}}\left(\mathcal{T}_{Z}>\lfloor t y / a\rfloor, \chi(y)>v\right)= \\
=\lim _{y \rightarrow+\infty} \widetilde{\mathbf{P}}\left(\frac{\mathcal{T}_{Z}-Z / a}{\sigma a^{-3 / 2} \sqrt{Z / t}}>\frac{\lfloor t y / a\rfloor-Z / a}{\sigma a^{-3 / 2} \sqrt{Z / t}}, \chi(y)>v\right)= \\
=\widehat{\mathbf{P}}(B(t)>-z) \widehat{\mathbf{P}}(\chi>v)=\widehat{\mathbf{P}}(B(t)<z) \widehat{\mathbf{P}}(\chi>v)
\end{gathered}
$$

Аналогично показывается, что

$$
\lim _{y \rightarrow+\infty} \widetilde{\mathbf{P}}\left(\mathcal{T}_{Z+l}>\lfloor t y / a\rfloor, \chi(y)>v\right)=\widehat{\mathbf{P}}(B(t)<z) \widehat{\mathbf{P}}(\chi>v) .
$$

Перейдем в соотношении (59) к пределу сначала при $y \rightarrow+\infty$, а затем при $l \rightarrow$ $+\infty$. Учитывая соотношения (60)-(62), получаем

$$
\lim _{y \rightarrow+\infty} \widetilde{\mathbf{P}}\left(V_{y}(t) \leqslant z, \chi(y)>v\right)=\widehat{\mathbf{P}}(B(t)<z) \widehat{\mathbf{P}}(\chi>v),
$$

что требовалось доказать.

Покажем, что при $y \rightarrow+\infty$

$$
\left\{U_{y}(t), t \in[0,1] \mid D_{x}\right\} \stackrel{\widetilde{D}}{\rightarrow} B .
$$

В силу соотношения (57) для произвольных моментов времени $0<t_{1}<t_{2}<\ldots<$ $t_{m} \leqslant 1$ и чисел $x_{1}, x_{2}, \ldots, x_{m}$, где $m \in \mathbb{N}$,

$$
\begin{aligned}
& \widetilde{\mathbf{P}}\left(V_{y}^{\left(k, \beta_{2}\right)}\left(t_{1}\right) \leqslant x_{1}, \ldots, V_{y}^{\left(k, \beta_{2}\right)}\left(t_{m}\right) \leqslant x_{m}, D_{x, k}\right) \leqslant \\
& \leqslant \widetilde{\mathbf{P}}\left(U_{y}\left(t_{1}\right) \leqslant x_{1}, \ldots, U_{y}\left(t_{m}\right) \leqslant x_{m}, D_{x, k}\right) \leqslant \\
& \leqslant \widetilde{\mathbf{P}}\left(V_{y}^{\left(k, \beta_{1}\right)}\left(t_{1}\right) \leqslant x_{1}, \ldots, V_{y}^{\left(k, \beta_{1}\right)}\left(t_{m}\right) \leqslant x_{m}, D_{x, k}\right)
\end{aligned}
$$

(при этом выбираем $y$ так, чтобы $\left.(k+1) a<t_{1} y\right)$. Сначала перейдем от $D_{x, k}$ к $G_{k}$ :

$$
\widetilde{\mathbf{P}}\left(V_{y}^{\left(k, \beta_{2}\right)}\left(t_{1}\right) \leqslant x_{1}, \ldots, V_{y}^{\left(k, \beta_{2}\right)}\left(t_{m}\right) \leqslant x_{m}, G_{k}\right)-\widetilde{\mathbf{P}}\left(G_{k} \triangle D_{x, k}\right) \leqslant
$$




$$
\begin{gathered}
\leqslant \widetilde{\mathbf{P}}\left(U_{y}\left(t_{1}\right) \leqslant x_{1}, \ldots, U_{y}\left(t_{m}\right) \leqslant x_{m}, D_{x, k}\right) \leqslant \\
\leqslant \widetilde{\mathbf{P}}\left(V_{y}^{\left(k, \beta_{1}\right)}\left(t_{1}\right) \leqslant x_{1}, \ldots, V_{y}^{\left(k, \beta_{1}\right)}\left(t_{m}\right) \leqslant x_{m}, G_{k}\right) .
\end{gathered}
$$

Заметим, что при $i \in\{1,2\}$

$$
\begin{gathered}
\widetilde{\mathbf{P}}\left(V_{y}^{\left(k, \beta_{i}\right)}\left(t_{1}\right) \leqslant x_{1}, \ldots, V_{y}^{\left(k, \beta_{i}\right)}\left(t_{m}\right) \leqslant x_{m}, G_{k}\right)= \\
=\widetilde{\mathbf{E}}\left(\widetilde{\mathbf{P}}_{k}\left(V_{y}^{\left(k, \beta_{i}\right)}\left(t_{1}\right) \leqslant x_{1}, \ldots, V_{y}^{\left(k, \beta_{i}\right)}\left(t_{m}\right) \leqslant x_{m}\right) ; G_{k}\right)= \\
=\widetilde{\mathbf{E}}\left(\left.\widetilde{\mathbf{P}}_{k}\left(V_{y}^{\left(k, b_{i}\right)}\left(t_{1}\right) \leqslant x_{1}, \ldots, V_{y}^{\left(k, b_{i}\right)}\left(t_{m}\right) \leqslant x_{m}\right)\right|_{b_{i}=\beta_{i}} ; G_{k}\right)= \\
=\widetilde{\mathbf{E}}\left(\left.\widetilde{\mathbf{P}}\left(V_{y}\left(t_{1}-\Delta t\right)+b_{i} \Delta x \leqslant x_{1}, \ldots, V_{y}\left(t_{m}-\Delta t\right)+b_{i} \Delta x \leqslant x_{m}\right)\right|_{b_{i}=\beta_{i}} ; G_{k}\right),
\end{gathered}
$$

где $\Delta t=k a y^{-1}, \Delta x=\sqrt{a}(\sigma \sqrt{y})^{-1}$. Откуда, применяя (58), по теореме о мажорируемой сходимости находим, что при $i \in\{1,2\}$

$$
\begin{gathered}
\lim _{y \rightarrow+\infty} \widetilde{\mathbf{P}}\left(V_{y}^{\left(k, \beta_{i}\right)}\left(t_{1}\right) \leqslant x_{1}, \ldots, V_{y}^{\left(k, \beta_{i}\right)}\left(t_{m}\right) \leqslant x_{m}, G_{k}\right)= \\
=\widehat{\mathbf{P}}\left(B\left(t_{1}\right) \leqslant x_{1}, \ldots, B\left(t_{m}\right) \leqslant x_{m}\right) \widetilde{\mathbf{P}}\left(G_{k}\right) .
\end{gathered}
$$

Перейдем к пределу в соотношении (64) сначала при $y \rightarrow+\infty$, а затем при $k \rightarrow \infty$. В силу соотношений (10), (65) и (27)

$$
\begin{gathered}
\lim _{k \rightarrow \infty} \lim _{y \rightarrow+\infty} \widetilde{\mathbf{P}}\left(U_{y}\left(t_{1}\right) \leqslant x_{1}, \ldots, U_{y}\left(t_{m}\right) \leqslant x_{m}, D_{x, k}\right)= \\
=\widehat{\mathbf{P}}\left(B\left(t_{1}\right) \leqslant x_{1}, \ldots, B\left(t_{m}\right) \leqslant x_{m}\right) \widetilde{\mathbf{P}}(D) .
\end{gathered}
$$

Теперь перейдем от $D_{x, k}$ к $D_{x}$ :

$$
\begin{gathered}
\widetilde{\mathbf{P}}\left(U_{y}\left(t_{1}\right) \leqslant x_{1}, \ldots, U_{y}\left(t_{m}\right) \leqslant x_{m}, D_{x, k}\right) \leqslant \\
\leqslant \widetilde{\mathbf{P}}\left(U_{y}\left(t_{1}\right) \leqslant x_{1}, \ldots, U_{y}\left(t_{m}\right) \leqslant x_{m}, D_{x}\right) \leqslant \\
\leqslant \widetilde{\mathbf{P}}\left(U_{y}\left(t_{1}\right) \leqslant x_{1}, \ldots, U_{y}\left(t_{m}\right) \leqslant x_{m}, D_{x, k}\right)+\widetilde{\mathbf{P}}\left(D_{x} \triangle D_{x, k}\right) .
\end{gathered}
$$

Откуда, переходя к пределу сначала при $y \rightarrow+\infty$, а затем при $k \rightarrow \infty$ и используя соотношения (10) и (66), получаем, что

$$
\begin{gathered}
\lim _{y \rightarrow+\infty} \widetilde{\mathbf{P}}\left(U_{y}\left(t_{1}\right) \leqslant x_{1}, \ldots, U_{y}\left(t_{m}\right) \leqslant x_{m}, D_{x}\right)= \\
=\widehat{\mathbf{P}}\left(B\left(t_{1}\right) \leqslant x_{1}, \ldots, B\left(t_{m}\right) \leqslant x_{m}\right) \widetilde{\mathbf{P}}(D) .
\end{gathered}
$$

Но это утверждение вместе с (26) означает, что

$$
\begin{gathered}
\lim _{y \rightarrow+\infty} \widetilde{\mathbf{P}}\left(U_{y}\left(t_{1}\right) \leqslant x_{1}, \ldots, U_{y}\left(t_{m}\right) \leqslant x_{m} \mid D_{x}\right)= \\
=\widehat{\mathbf{P}}\left(B\left(t_{1}\right) \leqslant x_{1}, \ldots, B\left(t_{m}\right) \leqslant x_{m}\right),
\end{gathered}
$$

т.е. сходимость конечномерных распределений в (63) установлена. 
Покажем, что для произвольного $\varepsilon^{\prime}>0$

$$
\lim _{\delta \rightarrow 0} \limsup _{k \rightarrow \infty} \limsup _{y \rightarrow+\infty} \widetilde{\mathbf{P}}\left(w_{Y_{x}}(\delta) \geqslant \varepsilon^{\prime} ; D_{x, k}\right)=0 .
$$

Если событие $D_{x, k}$ произошло, то, учитывая $(57)$ и $(30)$, находим, что при $t_{y}=$ $(k+1) a / y$

$$
w_{U_{y}}(\delta) \leqslant 2 \sup _{\left[t \in 0, t_{y}\right)}\left|U_{y}(t)\right|+w_{V_{y}^{\left(k, \beta_{1}\right)}}(\delta)+\sup _{t \in[0,1]}\left|V_{y}^{\left(k, \beta_{2}\right)}(t)-V_{y}^{\left(k, \beta_{1}\right)}(t)\right| .
$$

Заметим, что $w_{V_{y}^{(k, b)}}(\delta)$ не зависит от $b$. Кроме того,

$$
\sup _{t \in[0,1]}\left|V_{y}^{\left(k, \beta_{2}\right)}(t)-V_{y}^{\left(k, \beta_{1}\right)}(t)\right|=\frac{\beta_{2}-\beta_{1}}{\sigma \sqrt{y / a}}=\frac{\ln \left[(1+\varepsilon)(1-\varepsilon)^{-1}\right]}{\sigma \sqrt{y / a}} .
$$

Это означает, что если событие $D_{x, k}$ произошло, то

$$
w_{U_{y}}(\delta) \leqslant 2 \sup _{\left[t \in 0, t_{y}\right)}\left|U_{y}(t)\right|+w_{V_{y}^{(k, 0)}}(\delta)+\frac{\ln \left[(1+\varepsilon)(1-\varepsilon)^{-1}\right]}{\sigma \sqrt{y / a}} .
$$

Ввиду (68) для справедливости (67) достаточно показать, что

$$
\begin{gathered}
\lim _{\delta \rightarrow 0} \limsup _{k \rightarrow \infty} \limsup _{y \rightarrow+\infty} \widetilde{\mathbf{P}}\left(2 \sup _{\left[t \in 0, t_{y}\right)}\left|U_{x}(t)\right| \geqslant \frac{\varepsilon^{\prime}}{2}\right)=0, \\
\lim _{\delta \rightarrow 0} \limsup _{k \rightarrow \infty} \limsup _{y \rightarrow+\infty} \widetilde{\mathbf{P}}\left(w_{V_{y}^{(k, 0)}}(\delta) \geqslant \frac{\varepsilon^{\prime}}{2}\right)=0 .
\end{gathered}
$$

Нетрудно понять, что

$$
\sup _{t \in\left[0, t_{y}\right)}\left|U_{y}(t)\right| \leqslant \frac{\ln \sup _{t \in\left[0, t_{y}\right)} \xi_{\lfloor t y / a\rfloor}+t_{y} y}{\sigma \sqrt{y / a}}=\frac{\ln \sup _{i \leqslant k} \xi_{i}+(k+1) a}{\sigma \sqrt{y / a}},
$$

откуда следует (69). Далее,

$$
V_{y}^{(k, 0)}(t)=\frac{S_{(\lfloor t y / a\rfloor-k)^{+}}^{(k)}-t y}{\sigma \sqrt{y / a}}=\frac{S_{(\lfloor(t-\Delta t) y / a\rfloor)^{+}}^{(k)}-(t-\Delta t) y}{\sigma \sqrt{y / a}}-k a \Delta x .
$$

Значит, модуль непрерывности левой части не превосходит модуля непрерывности функции $\left(S_{\lfloor t y / a\rfloor}^{(k)}-t y\right)(\sigma \sqrt{y / a})^{-1}$ и

$$
\widetilde{\mathbf{P}}\left(w_{V_{y}^{(k, 0)}}(\delta) \geqslant \frac{\varepsilon^{\prime}}{2}\right) \leqslant \widetilde{\mathbf{P}}\left(w_{V_{y}}(\delta) \geqslant \frac{\varepsilon^{\prime}}{2}\right) .
$$

Вспоминая теперь (58), приходим к (70). Таким образом, соотношение (67) доказано.

Заметим, что

$$
\widetilde{\mathbf{P}}\left(w_{Y_{y}}(\delta) \geqslant \varepsilon^{\prime} ; D_{x}\right) \leqslant \widetilde{\mathbf{P}}\left(w_{Y_{y}}(\delta) \geqslant \varepsilon^{\prime} ; D_{x, k}\right)+\widetilde{\mathbf{P}}\left(D_{x} \triangle D_{x, k}\right) .
$$


Переходя в этом неравенстве к пределу сначала при $y \rightarrow+\infty$, затем при $k \rightarrow \infty$ и, наконец, при $\delta \rightarrow 0$ и учитывая соотношения (9), (67) и (26), получаем, что

$$
\lim _{\delta \rightarrow 0} \limsup _{y \rightarrow+\infty} \widetilde{\mathbf{P}}\left(w_{Y_{y}}(\delta) \geqslant \varepsilon^{\prime} \mid D_{x}\right)=0 .
$$

Это утверждение наряду со сходимостью конечномерных распределений означает справедливость соотношения (63).

Объединяя левые части соотношений (40) и (63) и повторяя рассуждения, использованные при их доказательстве, а также используя асимптотическую независимость при $u \in(0,1)$ случайных элементов $\left\{V_{y}(t), t \in[0, u]\right\}$ и $\chi(y)$ при $y \rightarrow+\infty$, можно доказать асимптотическую независимость процесса $\left\{U_{y}(t), t \in[0, u] \mid D_{x}\right\}$ и левой части соотношения (40).

Перейдем к завершающей части доказательства. Для $u \in(0,1)$ введем случайное событие

$$
\widehat{D}_{x}=\left\{\frac{u y}{a}<T_{x}<+\infty\right\},
$$

где $x=\exp (y)$. Заметим, что в силу закона больших чисел для $T_{x}$

$$
\lim _{x \rightarrow+\infty} \mathbf{P}\left(D_{x} \backslash \widehat{D}_{x} \mid D_{x}\right)=0 .
$$

Пусть $f$ - произвольная ограниченная непрерывная числовая функция, заданная на $D[0, u]$. Аналогично (51) устанавливается, что

$$
\mathbf{E}\left(f\left(U_{y}\right) ; \widehat{D}_{x}\right)=\widetilde{\mathbf{E}}\left(\exp \left(-\varkappa S_{T_{x}}\right) f\left(U_{y}\right) ; \widehat{D}_{x}\right),
$$

где $U_{y}=\left\{U_{y}(t), t \in[0, u]\right\}$. При этом используется измеримость случайной величины $f\left(U_{y}\right) I\left(T_{x}=m\right)$ относительно $\sigma$-алгебры $\sigma_{m}$ при $m>u y / a$. Поскольку $\widehat{D}_{x} \subset D_{x}$, из соотношений $(51)$ и $(72)$ (при $f \equiv 1$ ) вытекает, что

$$
\mathbf{P}\left(D_{x} \backslash \widehat{D}_{x}\right)=\widetilde{\mathbf{E}}\left(\exp \left(-\varkappa S_{T_{x}}\right) ; D_{x} \backslash \widehat{D}_{x}\right)
$$

Из соотношений (72), (73) находим, что

$$
\left|\mathbf{E}\left(f\left(U_{y}\right) ; D_{x}\right)-\widetilde{\mathbf{E}}\left(\exp \left(-\varkappa S_{T_{x}}\right) f\left(U_{y}\right) ; D_{x}\right)\right| \leqslant 2 K \mathbf{P}\left(D_{x} \backslash \widehat{D}_{x}\right) .
$$

где $K>0$ - постоянная, ограничивающая модуль функции $f$. Следовательно,

$$
\left|\mathbf{E}\left(f\left(U_{y}\right) \mid D_{x}\right)-\frac{\widetilde{\mathbf{E}}\left(\exp \left(-\varkappa S_{T_{x}}\right) f\left(U_{y}\right) ; D_{x}\right)}{\mathbf{P}\left(D_{x}\right)}\right| \leqslant 2 K \mathbf{P}\left(D_{x} \backslash \widehat{D}_{x} \mid D_{x}\right) .
$$

Заметим, что

$$
\widetilde{\mathbf{E}}\left(\exp \left(-\varkappa S_{T_{x}}\right) f\left(U_{y}\right) \mid D_{x}\right)=x^{-\varkappa} \widetilde{\mathbf{E}}\left(\left(\frac{\psi_{x}}{\varphi_{x}}\right)^{\varkappa} f\left(U_{y}\right) \mid D_{x}\right) .
$$

Из (40), (63) и асимптотической независимости процесса $\left\{U_{y} \mid D_{x}\right\}$ и левой части соотношения (40) следует, что при $y \rightarrow+\infty$

$$
\left\{\left(\frac{\psi_{x}}{\varphi_{x}}\right)^{\varkappa} f\left(U_{y}\right) \mid D_{x}\right\} \stackrel{\widetilde{D}}{\rightarrow}(\widehat{W})^{\varkappa} e^{-\varkappa \chi} f(B),
$$


причем семейство (при разных $x$ ) случайных величин в левой части равномерно интегрируемо. В итоге получаем, что

$$
\lim _{y \rightarrow+\infty} \widetilde{\mathbf{E}}\left(\left(\frac{\psi_{x}}{\varphi_{x}}\right)^{\varkappa} f\left(U_{y}\right) \mid D_{x}\right)=\widetilde{\mathbf{E}} W^{\varkappa} \widehat{\mathbf{E}} e^{-\varkappa \chi} \widehat{\mathbf{E}} f(B) .
$$

Из соотношений (75)-(76) вытекает, что при $y \rightarrow+\infty$

$$
\widetilde{\mathbf{E}}\left(\exp \left(-\varkappa S_{T_{x}}\right) f\left(U_{y}\right) ; D_{x}\right) \sim x^{-\varkappa} \widetilde{\mathbf{E}} W^{\varkappa} \widehat{\mathbf{E}} e^{-\varkappa \chi} \widehat{\mathbf{E}} f(B)
$$

и (см. замечание 1)

$$
\lim _{y \rightarrow+\infty} \widetilde{\mathbf{E}}\left(\exp \left(-\varkappa S_{T_{x}}\right) f\left(U_{y}\right) ; D_{x}\right) / \mathbf{P}\left(D_{x}\right)=\widehat{\mathbf{E}} f(B) .
$$

Переходя в соотношении (74) к пределу при $y \rightarrow+\infty$ и учитывая соотношения (71) и $(77)$, получаем, что

$$
\lim _{y \rightarrow+\infty} \mathbf{E}\left(f\left(U_{y}\right) ; D_{x}\right)=\widehat{\mathbf{E}} f(B),
$$

что завершает доказательство теоремы 2.

\section{Список литературы}

1. Afanasyev V. I.,Geiger J., Kersting G., Vatutin V. A., "Criticality for branching processes in random environment", Ann. Probab., 33:2 (2005), 645-673.

2. Afanasyev V. I., "On the maximum of a subcritical branching process in a random environment", Stochastic Process. Appl., 93:1 (2001), 84-107.

3. Афанасьев В. И., "Высокоуровневые докритические ветвящиеся процессы в случайной среде", Тр. МИАН, 282 (2013), 10-21.

4. Афанасьев В. И., "Принцип инвариантности для критического ветвящегося процесса в случайной среде, достигающего высокого уровня", Теория вероятн. и ее примен., 54:1 (2009), 3-17.

5. Afanasyev V. I., "New invariance principles for critical branching process in random environment", Advances in data analysis. Stat. Ind. Technol., 2010, 105-115.

6. Афанасьев В. И., "Броуновский прыжок в высоту", Теория вероятн. и ее примен., 55:2 (2010), 209-225.

7. Gut A., Stopped random walks, New York: Springer-Verlag, 1988.

8. Siegmund D., Sequential analysis tests and confidence intervals, New York: Springer, 1985.

9. Биллингсли П., Сходимость вероятностных мер, М.: Наука, 1977.

Статья поступила 26.12.2013. 